\title{
A CHARACTERIZATION OF PLANE LIGHT OPEN MAPPINGS
}

\author{
M. K. FORT, JR.
}

1. Minimal functions. We are concerned with continuous functions whose domains are open subsets of the plane $P$ and whose ranges are contained in $P$. In this paper the word mapping is used to designate such a function. By a disk we mean a subset of $P$ which is a closed topological 2-cell. If $S$ is a simple closed curve in $P$, we denote by $S^{*}$ the disk formed by taking the union of $S$ and the interior of $S$.

Let $S$ be a simple closed curve in $P$ and let $f$ be a mapping whose domain contains $S^{*}$. We say that $f$ is minimal on $S^{*}$ if $f\left(S^{*}\right) \subset g\left(S^{*}\right)$ for every mapping $g$ whose domain contains $S^{*}$ and which is such that $f|S=g| S$ (that is, which is such that $f(x)=g(x)$ for each $x \in S$ ). We define $f$ to be minimal if $f$ is minimal on each disk contained in the domain of $f$.

In this paper we prove that a light mapping is open if and only if it is minimal.

2. Winding numbers. We make use of the concept of winding number or topological index. If $f$ is a mapping whose domain contains a simple closed curve $S$ and $p \in P-f(S)$, then we denote by $W(f, S, p)$ the winding number of $f$ on $S$ with respect to $p$. Intuitively, as a point $x$ travels once around $S$ in a counter-clockwise direction, $W(f, S, p)$ is the net number of revolutions that the vector from $p$ to $f(x)$ makes about $p$ (a revolution being positive if made in a counter-clockwise direction and being negative if made in a clockwise direction).

The following facts concerning winding numbers are well known and are assumed.

(i) If $p \in P-f(S)$, then there exists a neighborhood $V$ of $p$ such that $W(f, S, v)=W(f, S, p)$ for each $v \in V$.

(ii) If $p \in P-f(S)$, then $f \mid S$ is homotopic in $P-p$ to a constant if and only if $W(f, S, p)=0$. $(f \mid S$ is the function obtained by restricting the domain of $f$ to $S$.)

(iii) If $p \in P-f(S)$, then $f \mid S$ can be extended to a continuous function on $S^{*}$ into $P-p$ if and only if $W(f, S, p)=0$.

(iv) If the domain of a mapping $f$ contains a disk $S^{*}$, then $f\left(S^{*}\right)$ $\supset f(S) \cup\{p \mid W(f, S, p) \neq 0\}$.

(v) If $S_{1}$ and $S_{2}$ are simple closed curves which have disjoint interiors, $S_{1} \cap S_{2}$ is an arc, and

Received by the editors February 6, 1950. 


$$
S=\mathrm{Cl}\left(\left(S_{1} \cup S_{2}\right)-\left(S_{1} \cap S_{2}\right)\right)
$$

then

$$
W(f, S, p)=W\left(f, S_{1}, p\right)+W\left(f, S_{2}, p\right)
$$

for each $p \in P-\left(S_{1} \cup S_{2}\right)$.

THEOREM 1. If $S$ is a simple closed curve and $f$ is a mapping whose domain contains $S^{*}$, then $f$ is minimal on $S^{*}$ if and only if

$$
f\left(S^{*}\right)=f(S) \cup\{p \mid W(f, S, p) \neq 0\} .
$$

Let $g$ be a mapping on $S^{*}$ for which $g|S=f| S$. It follows from (iii) that if $p \in P-f(S)$ and $W(f, S, p) \neq 0$, then $p \in g\left(S^{*}\right)$. Thus, if (A) holds, then $f\left(S^{*}\right) \subset g\left(S^{*}\right)$ and $f$ is minimal on $S^{*}$.

If (A) does not hold, then it follows from (iv) that there exists $p \in f\left(S^{*}\right)-f(S)$ for which $W(f, S, p)=0$. By (iii), there exists an extension $g$ of $f \mid S$ such that $g\left(S^{*}\right) \subset P-p$. It follows that $f\left(S^{*}\right) \nsubseteq g\left(S^{*}\right)$ and hence that $f$ is not minimal on $S^{*}$.

3. Plane light open mappings. A mapping is open (or strongly interior) if it takes open sets into open sets. A mapping $f$ is light if $f^{-1}(y)$ is totally disconnected for each $y$ in the range of $f$.

Theorem 2. A light mapping $f$ is open if and only if it is minimal.

Suppose that $f$ is light and minimal. Let $U$ be an open subset of the domain of $f$, and let $y \in f(U)$. Choose $x \in U$ so that $f(x)=y$. Since $f^{-1}(y)$ is totally disconnected, it can be shown that there exists a simple closed curve $S$ in $U-f^{-1}(y)$ such that $x \in S^{*} \subset U$. Since $y \notin f(S), W(f, S, y)$ is defined. Since $y \in f\left(S^{*}\right)-f(S)$ and $f$ is minimal, it follows from Theorem 1 that $W(f, S, y) \neq 0$. It follows from (i) that there is a neighborhood $V$ of $y$ such that $W(f, S, v) \neq 0$ for each $v \in V$. Now, from (iv), we obtain $V \subset f(U)$. Thus $f(U)$ is an open set and $f$ is an open mapping.

Now suppose that $f$ is light and open. Let $S$ be a simple closed curve for which $S^{*}$ is contained in the domain of $f$ and let $y \in f\left(S^{*}\right)-f(S)$. It is known (see $[1, \text { p. 191]) })^{1}$ that $f^{-1}(y) \cap S^{*}$ is a finite set. We let $x_{1}, \cdots, x_{n}$ be the points of $f^{-1}(y) \cap S^{*}$. Locally at each $x_{i}, f$ is topologically equivalent to a mapping $f_{i}$ on the disk $\{z \mid z \in P$ and $|z| \leqq 1\}$, where $f_{i}(z)=z^{k_{i}}$ and $k_{i}$ is a positive integer. (In this connection see $[1$, p. 198].) It is now easy to see that for each $i$ there is a simple closed curve $C_{i}$ about $x_{i}$ for which $C_{i}{ }^{*} C S^{*}$ and $\left|W\left(f, C_{i}, y\right)\right|=k_{i}$. Thus the integers $W\left(f, C_{i}, y\right)$ are all nonzero. Interior to $C_{i}$ there exist

${ }^{1}$ Numbers in brackets refer to the bibliography at the end of the paper. 
points at which $f$ is locally a homeomorphism. At such points $f$ is orientation preserving if $W\left(f, C_{i}, y\right)$ is positive and orientation reversing if $W\left(f, C_{i}, y\right)$ is negative. The set $L$ of points of $S^{*}$ at which $f$ is locally a homeomorphism is a dense connected subset of $S^{*}$. The subset $M$ of $L$ consisting of all points at which $f$ is orientation preserving is both open and closed relative to $L$. Hence $M=L$ or $M$ is the empty set. It follows that the integers $W\left(f, C_{i}, y\right)$ are either all positive or all negative. We may obviously choose the curves $C_{i}$ so that the disks $C_{i}^{*}$ are disjoint and do not intersect $S$, and we assume that this has been done. We now choose a finite cellular subdivision of $S^{*}$ in such a way that each $C_{i}^{*}$ is a 2 -cell of the subdivision. We let $A_{1}, \cdots, A_{m}$ be the 2-cells of the subdivision and let $B_{j}$ be the boundary of $A_{j}$. Making use of (v) and induction, we obtain

$$
W(f, S, y)=\sum_{j=1}^{m} W\left(f, B_{j}, y\right) .
$$

Unless $B_{j}=C_{i}$ for some $i$, then $y \notin f\left(B_{j}^{*}\right)$ and $W\left(f, B_{j}, y\right)=0$ by (iii). Therefore

$$
W(f, S, y)=\sum_{j=1}^{m} W\left(f, B_{j}, y\right)=\sum_{i=1}^{n} W\left(f, C_{i}, y\right) .
$$

Since the numbers $W\left(f, C_{i}, y\right)$ are all nonzero and are of like sign, we obtain $W(f, S, y) \neq 0$. It now follows from Theorem 1 that $f$ is minimal.

It would be interesting to know whether or not theorems analogous to Theorem 2 exist for higher-dimensional spaces. The author has been unable to prove such theorems.

\section{BibliograPHy}

1. G. T. Whyburn, Analytic topology, Amer. Math. Soc. Colloquium Publications, vol. 28, New York, 1942.

UNIVERSITY OF ILLINOIS 